\title{
Functional consequences of $\mathrm{C} 5$ nephritic factors in $\mathrm{C} 3$ glomerulopathies
}

C3 glomerulopathies (C3G) are caused by dysregulation of the alternative complement pathway. In $>50 \%$ of cases, these diseases are associated with expression of autoantibodies known as $\mathrm{C} 3$ nephritic factors (C3Nefs), which stabilize the alternative pathway $\mathrm{C} 3$ convertase $\mathrm{C} 3 \mathrm{bBb}$. Now, researchers report a role of C5Nefs, which stabilize the C5 convertase, in determining the biological phenotype of C3G.

"In patients with C3Nefs, the clinical presentation and biomarker profiles of $\mathrm{C} 3$ and C5 convertases are very heterogeneous, suggesting different biological effects of Nefs on convertase stabilization," explains MariaChiara Marinozzi. "Our aim was to better understand the biological effects of Nefs on convertases as well as the role of properdin in C3Nef-mediated convertase stabilization."

The researchers identified C3Nefs in 75\% and C5Nefs in 45\% of IgG samples from 101 patients with C3G. They found that the capacity of purified patient IgG to stabilize C5 convertase, but not $\mathrm{C} 3 \mathrm{bBb}$, correlated with the plasma levels of the terminal complement complex sC5b-9, indicating dysregulation of the $\mathrm{C} 5$ convertase. Properdin enhanced the stabilization of $\mathrm{C} 3 \mathrm{bBb}$ in the presence of IgG that was positive for both C3Nefs and C5Nefs, but did not have such an effect in the presence of IgG that was positive for C3Nefs alone. Analysis of patient biopsy samples and autoantibody profiles suggested that $\mathrm{C} 5 \mathrm{Nef}$ expression was associated with C3 glomerulonephritis rather than dense deposit disease.

The researchers conclude that C3Nefs and C5Nefs have distinct functional consequences and pathological associations. They suggest that prevention of $\mathrm{C} 5$ activation or inhibition of properdin binding to $\mathrm{C} 3 \mathrm{bBb}$ could be potentially beneficial therapeutic strategies for C5Nef-positive patients with C3G.

Ellen F. Carney

ORIGINAL ARTICLE Marinozzi, M.-C.et al. C5 nephritic

factors drive the biological phenotype of $\mathrm{C} 3$ glomerulopathies. Kidney Int. http://dx.doi.org/10.1016/j.kint.2017.04.017 (2017) ce

C5Nef expression

was associated

with C3

glomerulonephritis rather than dense

deposit disease 